\title{
Self-Induced Faraday Instability Laser
}

\author{
A. M. Perego, ${ }^{1, *}$ S. V. Smirnov, ${ }^{2}$ K. Staliunas, ${ }^{3,4}$ D. V. Churkin, ${ }^{2}$ and S. Wabnitz ${ }^{2,5}$ \\ ${ }^{1}$ Aston Institute of Photonics Technologies, Aston University, Aston Express Way, B4 7ET Birimingham, United Kingdom \\ ${ }^{2}$ Novosibirsk State University, 1 Pirogova str., Novosibirsk 630090, Russia \\ ${ }^{3}$ Departament de Fisica i Enginyeria Nuclear, Universitat Politècnica de Catalunya, E-08222 Barcelona, Spain \\ ${ }^{4}$ Institució Catalana de Recerca i Estudis Avançats, Passeig Lluis Companys 23, E-08010 Barcelona, Spain \\ ${ }^{5}$ Dipartimento di Ingegneria dell'Informazione, Università di Brescia, \\ and Istituto Nazionale di Ottica-Consiglio Nazionale delle Ricerche, Via Branze 38, Brescia 25123, Italy
}

(Received 20 December 2017; published 22 May 2018)

\begin{abstract}
We predict the onset of self-induced parametric or Faraday instabilities in a laser, spontaneously caused by the presence of pump depletion, which leads to a periodic gain landscape for light propagating in the cavity. As a result of the instability, continuous wave oscillation becomes unstable even in the normal dispersion regime of the cavity, and a periodic train of pulses with ultrahigh repetition rate is generated. Application to the case of Raman fiber lasers is described, in good quantitative agreement between our conceptual analysis and numerical modeling.
\end{abstract}

DOI: 10.1103/PhysRevLett.120.213902

Introduction.-In this Letter, we propose and theoretically analyze a new mechanism for pulse train and frequency comb generation in lasers, based on parametric (or Faraday) instability. Parametric instabilities (PIs) are a particular class of the more general family of modulation instabilities (MIs). MI is a universal mechanism that leads to the breakup of continuous waves (cw's) into modulated patterns in weakly dispersive and nonlinear physical systems. MI-activated pattern formation has been observed in a variety of different systems, from hydrodynamics to plasmas and optics [1-3]. Transverse pattern formation in lasers, which has been actively studied since late 1980s, is also due to MI leading to light patterns in the transverse direction to optical axis of the laser [4-7]. In the case of single-transverse, multilongitudinal mode lasers, e.g., fiber lasers, MI can occur along the laser cavity and break the stability of $\mathrm{cw}$ oscillation. Hence it may permit the generation of a continuous train of pulses with ultrahigh repetition rates [8], which is of great interest in many applications, from optical metrology to communications. The initial proposal to achieve a MI laser was based on the synchronous and coherent injection of an intense laser beam into a passive optical fiber ring cavity [8-11]. In this way, MI-induced pulse train generation in a nonlinear dispersive cavity results in the formation of a coherent or mode-locked frequency comb. Even in the absence of an external pump laser, for sufficiently high-level pumping of the active medium in the cavity, MI may lead to the spontaneous transition from $\mathrm{cw}$ to pulsed operation, leading to the so-called "self-induced MI laser" [12,13]. Stabilization of the laser repetition rate and simultaneous substantial reduction of the laser threshold can be achieved by inserting a frequency-periodic linear filter in the active nonlinear cavity $[14,15]$. It has been observed that in this case the mechanism leading to pulse train formation can be more conveniently described in terms of a dissipative fourwave mixing process, which leads to self-induced pulse train formation, irrespective of the sign of the cavity group velocity dispersion [16-18]. Another paradigmatic example of MI, leading to spontaneous mode locking in lasers, is the Risken-Nummedal-Graham-Haken instability, which is induced by coherent Rabi splitting of the gain curve in correspondence to a second laser threshold [19-22].

Parametric instabilities are a well-known universal mechanism for pattern formation in many different branches of physics [23]. PIs in nonlinear and dispersive wave propagation occur whenever one of the medium parameters is periodically modulated along the longitudinal direction. For externally periodically forced systems, PI is also commonly known as Faraday instability, following the initial Faraday's observation of pattern formation induced by the modulation of the vertical position of an open fluid tank [24]. Besides hydrodynamics, Faraday-like patterns are observed in a wide range of physical settings, from crystallization dynamics, chemical systems, and optics [25-29]. In the context of fiber lasers and externally driven passive fiber resonators, it has been proposed that Faraday instability can lead to the generation of pulse trains when the cavity dispersion is periodically modulated [30-34].

In addition to parametrically forced systems, there are many physical systems which feature the presence of collective oscillations, and thus may spontaneously lead to parametric or Faraday instabilities. Consider for example Bose-Einstein condensates, where PI may be introduced by the harmonic modulation of the nonlinear interaction or the profile of the trapping potential [35-37], and nonlinear 
graded index multimode optical fibers, where a selfinduced intensity grating results from beam self-imaging [38]. Here we point out that a laser is a fundamental physical system where a periodic modulation of the gain naturally occurs in the cavity in the presence of pump depletion (see Fig. 1). The resulting Faraday instability is analogous to the PI that has been predicted in periodically amplified fiber optic communication links [39]. However, in a laser the wave dynamics is much more complex, as it requires consideration of the effects of finite gain bandwidth (or temporal diffusion) and nonlinear gain saturation, and it can be described in terms of the complex GinzburgLandau equation (CGLE) [40]. Note that a sideband instability also occurs in soliton lasers, but in that case the generation of sidebands is of a different nature, since it is due to the coupling between the soliton and dispersive waves [41-43].

Self-induced Faraday instability in the CGLE.-As we have already mentioned in the introduction, our goal is to characterize the self-induced Faraday instability where a parametric modulation arises spontaneously from the solutions of a nonlinear system - the laser-and it is not imposed from the external world. However, we first

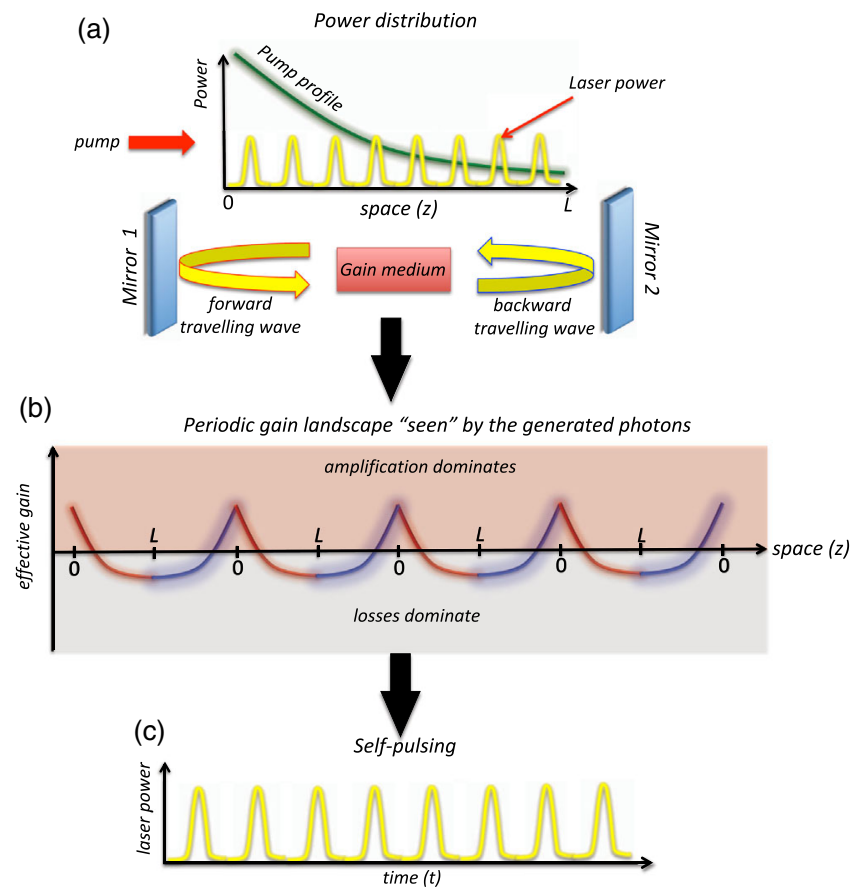

FIG. 1. Concept of self-induced Faraday instability laser: The spatially inhomogeneous gain profile naturally arising from the solutions of the nonlinear laser equations in the presence, for instance, of pump field injection from one side of the cavity (which is a typical situation in Raman fiber lasers) (a) gives rise to an effective periodic gain (and consequently nonlinearity) landscape profile (b) seen by the generated photons that travel back and forth in the linear cavity. Such a periodic gain and nonlinearity variations result in a parametric forcing leading to selfpulsing with high repetition rate (c). illustrate the generic features of the dynamical instability and of the associated pattern formation in the simplified and idealized case of the CGLE, while in the second part of this Letter we will provide a specific example based on numerical simulations of a realistic Raman fiber laser.

We have considered the following complex CGLE for the field envelope $A(z, t)$, defined in the local time reference frame $t$ and evolving along the spatial coordinate $z$ :

$$
\frac{\partial A}{\partial z}=\mu(z) A+(b-i d) \frac{\partial^{2} A}{\partial t^{2}}+(i c-s)|A|^{2} A .
$$

The nonlinearity coefficients describe self-phase modulation $c$ and gain saturation $s$, respectively, while $d$ and $b$ describe dispersion and finite gain bandwidth (diffusion).

Across our study, we have taken all coefficients with positive values, (Benjamin-Feir stable regime). The spatial dependent gain coefficient mimics the periodic gain profile experienced by the electric field upon propagation. The modulated gain coefficient is $\mu(z)=\mu_{\mathrm{av}}+\delta_{\mu} \cos \left(k_{m} z\right)$ with $\mu_{\mathrm{av}}$ equal to the average gain; the modulation occurs with modulation depth $\delta_{\mu}$ and with spatial period $L_{m}$ which defines the modulation wave number $k_{m}=2 \pi / L_{m}$. Such choice of the modulated gain coefficient is equivalent to "unfolding" the cavity for a light wave traveling back and forth in the resonator. In principle, also a ring cavity configuration would be possible, but it will not change the concept significantly, and furthermore the linear cavity may be much more easily realized experimentally. Of course, a purely sinusoidal modulation of the gain is in a certain sense an idealization, but still it is useful for a first characterization of the system behavior. A sinusoidal modulation in fact is the first and main harmonic of the periodic (unfolded) pump profile. In the final part of this Letter we will analyze in detail the Raman laser case where the naturally arising modulation of the pump profile will have a more "crestlike" shape.

Since we are dealing with a periodic system, a Floquet analysis allowed us to characterize the instability showing which modes experience amplification due to periodic forcing [29].

For each mode, we have defined the instability power gain as $G=2 \ln \left(\left|\lambda_{m}\right|\right) / L_{m}$, where $\lambda_{m}$ is the mode's Floquet multiplier with the largest absolute value. Instability takes place when $G>0$. Since the Floquet spectrum is symmetric with respect to frequency, throughout the Letter we have plotted only the positive frequency side.

For large enough $\delta_{\mu}$, we have observed a destabilization of the cw solution with net growth of modulation modes. A dependence of the instability gain on relevant parameters of the system is summarized in Fig. 2.

As one can expect, an increase in the forcing strength results in a broader synchronization region, which is typical for parametric resonances. Furthermore, together with the appearance of low frequency instability tongues which dominate the dynamics, we observe the presence 


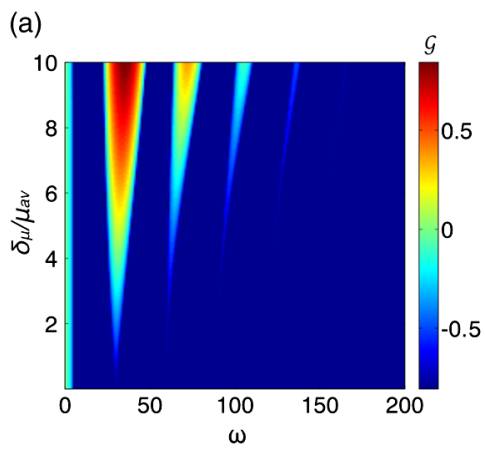

(b)

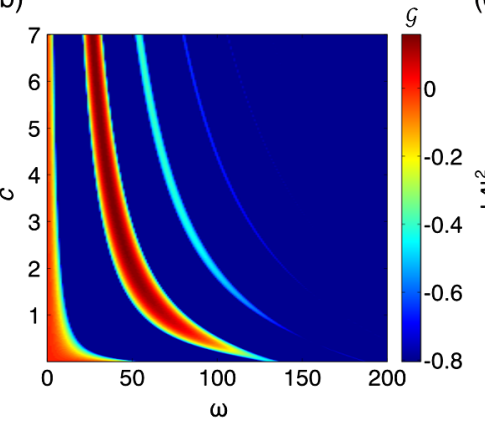

(c)

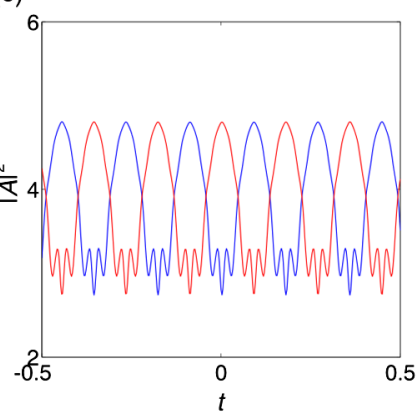

FIG. 2. Periodic variation of the gain induces MI when the modulation depth $\delta_{\mu}$ crosses a certain threshold: MI develops with the synchronization area increasing with $\delta_{\mu}$. In (a) the instability gain $G$ is plotted in the $\omega-\delta_{\mu} / \mu_{a v}$ plane. The instability frequency decreases with nonlinearity $c$, (b) hence showing that we are in the presence of a Faraday instability. Pattern formation: in (c) the stable temporal pattern, i.e., pulses on the finite background, is depicted. Blue and red lines correspond to the field modulus squared plotted at even (blue) and odd (red) modulation periods, respectively, after a long spatial evolution when the system has entered an asymptotic stable state. Common parameters to all plots are $c=5 s=0.1 d=1.18 \times 10^{-4}, \mu_{\mathrm{av}}=0.4, \delta_{\mu}=5 \mu_{\mathrm{av}}, L_{m}=1.5, b=0$; except for the case in (a) where $\delta_{\mu}$ varies from 0 to $10 \mu_{\mathrm{av}}$ and in (b) where $c$ varies from 0 to 7.

of underdeveloped instability tongues $(G<0)$, which correspond to higher-order parametric resonances.

A common feature of parametric instabilities in the normal dispersion or diffraction regime is the inverse scaling of the instability frequency versus nonlinearity. This trend is confirmed in our study [see Fig. 2(b)]. The growth of modulation unstable modes eventually results in a stable pattern formation. Here, similarly to other studies on parametric instabilities [31], but also as recently observed in semiconductor lasers [44], we are in the presence of a period- 2 dynamics of the pattern, corresponding to even and odd numbers of the modulation period, respectively [Fig. 2(c)].

It is indeed not surprising to recover here, at least qualitatively, the typical features of parametric instabilities, which in general are induced by the periodic modulation of dispersion and nonlinearity. In fact, the gain modulation considered automatically affects the nonlinearity, which leads to an effective spatially periodic modulation of the self-phase modulation coefficient $c$.
In the case of parametric instabilities, the first excited temporal mode has angular frequency $\omega_{\text {inst }}$ given by [29]

$$
\omega_{\mathrm{inst}} \approx \frac{\pi}{L_{m} \sqrt{2 c d \mu_{\mathrm{av}} / s}}
$$

The resemblance remains here, however, on a qualitative level, and Eq. (2) can be just considered as a rule of thumb, since the more involved nature of the dissipative modulation does not allow us to stretch the analogy further.

Coexistence of spatiotemporal chaos and Faraday patterns.-Achieving pulses that are stable and robust is a highly required feature of mode-locked lasers. Hence, understanding the impact of lasers' parameters on the pulses stability is of paramount importance. In this respect, we have preliminarily investigated the impact of the diffusion coefficient $b$ (gain bandwidth) on the stability of the self-induced Faraday pulses.

It is a widespread belief that losses play a stabilizing role in pattern formation processes. Surprisingly enough, as
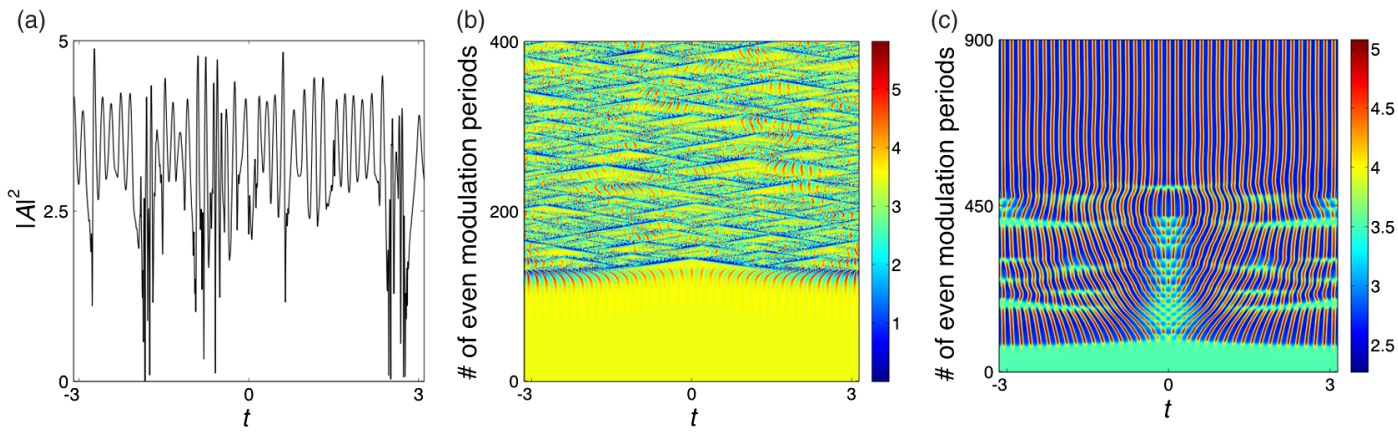

FIG. 3. In (a) we depict a typical example of a turbulent temporal intensity dynamics where periodic oscillations can still be observed. In (b) the spatiotemporal evolution of $|A|^{2}$ shows, in the presence of diffusion ( $b$ different from zero), the coexistence of spatiotemporal chaos and parametric patterns (high intensity red stripes); (a) is a section of (b). In (c) by reducing diffusion we obtain a stable pulse train. Parameters used in (a) and (b) are $c=5 s=0.2 d=1.18 \times 10^{-4}, \mu_{\mathrm{av}}=0.8, \delta_{\mu}=5 \mu_{\mathrm{av}}, L_{m}=1.5, b=1.97 \times 10^{-5}$. Plot (c) is obtained with the same parameters as (a) but with $b=0$. 
illustrated in Fig. 3, we observe that this is not the case for the particular situation that we are considering. Indeed, numerical simulations of Eq. (1) in the presence of diffusion show that parametric forcing destabilizes the homogeneous solution, leading to the onset of spatiotemporal chaos. First of all, Faraday patterns are excited; however, they are dynamically unstable [for the particular choice of the parameters used in Figs. 3(a) and 3(b)]. As a consequence, these patterns rapidly breakup, leading to a well-known scenario, which is typical for spatiotemporal extended chaotic systems [40,4547]. Nevertheless, we notice the interesting presence of Faraday pattern "islands" on a "sea" of spatiotemporally chaotic turbulence [see Figs. 3(a) and 3(b)]. If diffusion is reduced to below $b=10^{-6}$, we surprisingly observe that the laser dynamics is dominated by stable Faraday patterns [Fig. 3(c)] without any signature of turbulence. These considerations may have a relevant impact towards the practical design of Faraday instability lasers.

Self-induced Faraday instability in a fiber Raman laser.-As a proof of concept, we show by numerical simulations that self-induced Faraday instability owing to periodic gain variation can indeed be observed in a realistic laser system. We have considered here the all-normal dispersion linear cavity Raman fiber laser.

The pump field is injected at one cavity mirror, while the Stokes is generated spontaneously through the Raman scattering process along the fiber. Linear fiber attenuation together with pump depletion lead to a longitudinally dependent pump profile along the fiber [Fig. 4(a)]. The pump "modulation depth" becomes progressively pronounced with increasing values of its power. The pump field is most intense near one cavity mirror, while it is much weaker in the vicinity of the other one. Hence the Stokes field, while traveling back and forth in the cavity "experiences" both a periodic gain and a periodic nonlinearity profile. When the gain variation is sufficiently large, the threshold for parametric instability is crossed, and the amplification of spectral sidebands takes place. As a consequence, the $\mathrm{cw}$ solution of the cavity loses stability. Correspondingly, a pulse train is generated, with a repetition rate dictated by the instability frequency.

Light evolution in the laser is described by a set of four coupled generalized nonlinear Schrödinger equations for the forward and backward propagating fields $A_{p, s}^{ \pm}$, respectively [48], where suffixes $p$ and $s$ refer to the pump and Stokes fields:

$$
\begin{aligned}
\pm \frac{\partial A_{p}^{ \pm}}{\partial z}= & -\beta_{1 p} \frac{\partial A_{p}^{ \pm}}{\partial t}-i \frac{\beta_{2 p}}{2} \frac{\partial^{2} A_{p}^{ \pm}}{\partial t^{2}}-\frac{\alpha_{p}}{2} A_{p}^{ \pm}+i \gamma_{p}\left|A_{p}^{ \pm}\right|^{2} A_{p}^{ \pm} \\
& -\frac{g_{p}}{2}\left(\left|A_{s}^{ \pm}\right|^{2}+\left\langle\left|A_{s}^{\mp}\right|^{2}\right\rangle\right) A_{p}^{ \pm} \\
\pm \frac{\partial A_{s}^{ \pm}}{\partial z}= & -i \frac{\beta_{2 s}}{2} \frac{\partial^{2} A_{s}^{ \pm}}{\partial t^{2}}-\frac{\alpha_{s}}{2} A_{s}^{ \pm}+i \gamma_{s}\left|A_{s}^{ \pm}\right|^{2} A_{s}^{ \pm} \\
& +\frac{g_{s}}{2}\left(\left|A_{p}^{ \pm}\right|^{2}+\left\langle\left|A_{p}^{\mp}\right|^{2}\right\rangle\right) A_{s}^{ \pm} .
\end{aligned}
$$
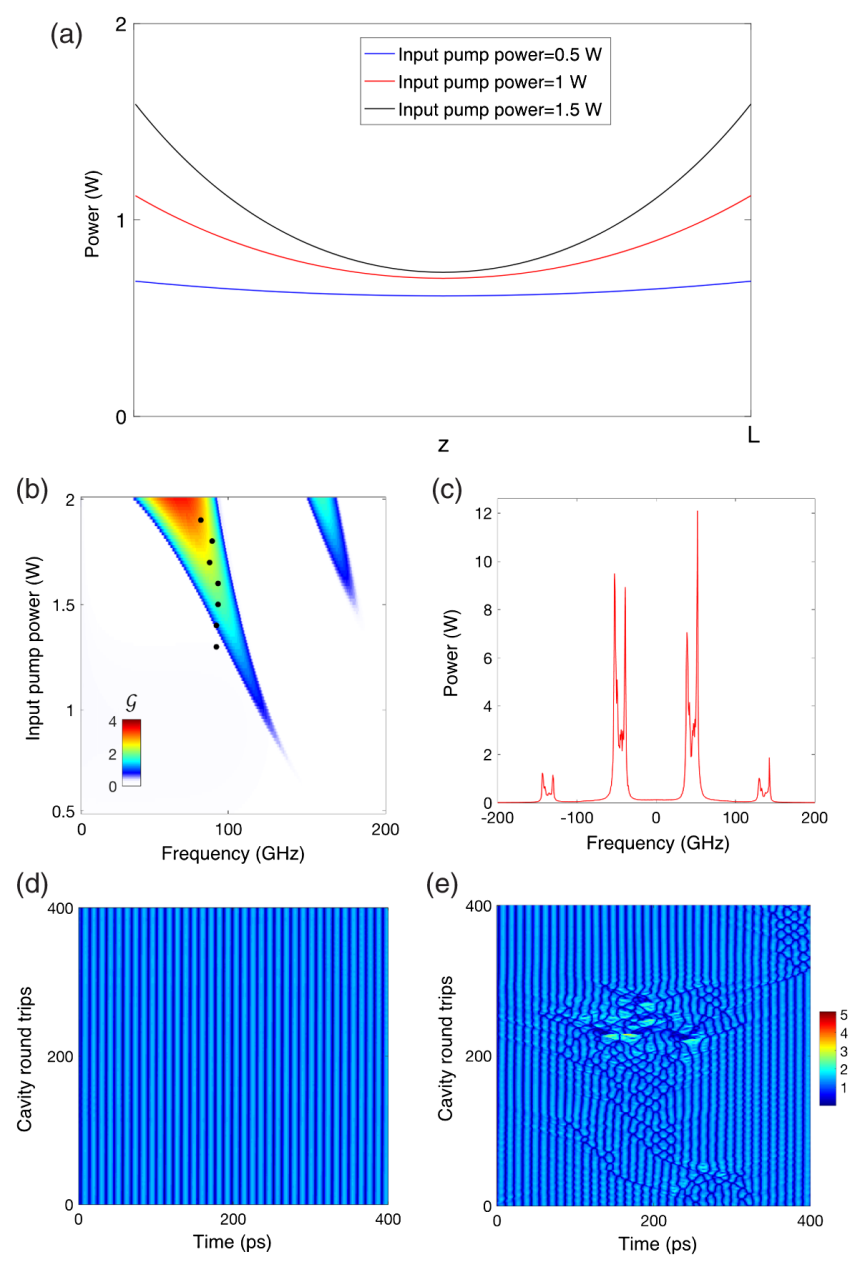

FIG. 4. In (a) the total pump power "experienced" by the Stokes field in the stationary state is plotted versus the longitudinal coordinate, for a length corresponding to one cavity round trip, and for various input pump power values. In (b) the Faraday instability gain $G$ is plotted versus pump power and frequency: colored areas correspond to unstable modes. (black dots denote generated pulses repetition rate). In (c) an example of optical spectrum is plotted for an input pump power equal to $1.3 \mathrm{~W}$. In (d) and (e), examples of stable and metastable pulses for input pump power equal to $1.3 \mathrm{~W}$ are depicted. Parameters used are $\gamma_{p}=3(\mathrm{Wkm})^{-1}, \gamma_{s}=2.57(\mathrm{Wkm})^{-1}, g_{p}=1.51(\mathrm{Wkm})^{-1}, g_{s}=$ $1.3(\mathrm{~W} \mathrm{~km})^{-1}, \alpha_{s}=0.8 \mathrm{~km}^{-1}, \alpha_{p}=0.5 \mathrm{~km}^{-1}$, fiber length $L=0.37 \mathrm{~km}$.

Here $\gamma_{p, s}, \beta_{2 p, s}, \alpha_{p, s}$, and $g_{p, s}$ denote the Kerr nonlinearity coefficient, group velocity dispersion, attenuation, and Raman gain for the pump and Stokes wavelengths, respectively, while $\beta_{1 p}$ describes the group velocity mismatch and the bra-kets denote temporal average. Equations (3) have been solved iteratively for forward and backward propagation with the following boundary conditions relating successive iterations:

$$
\tilde{A}_{s}^{-}(z=L)=f \tilde{A}_{s}^{+}(z=L), \tilde{A}_{s}^{+}(z=0)=f \tilde{A}_{s}^{-}(z=L),
$$

$\tilde{A}_{p}^{-}(z=L)=f \tilde{A}_{p}^{+}(z=L), \tilde{A}_{p}^{+}(z=0)=\sqrt{P} \delta(\omega)$, where $\sim$ denotes Fourier transformation, $f$ is a filter function with 
super-Gaussian order 3 shape, $1 \mathrm{~nm}$ FWHM for Stokes and 0.2 for pump, $P$ is the input pump power, and $\delta(\omega)$ the Dirac delta-function. The pump and Stokes wavelengths are 1060 and $1234 \mathrm{~nm}$, respectively.

The stability properties of the homogeneous solution of the Raman laser can be characterized once again by means of a Floquet linear stability analysis, whose results are depicted in Fig. 4(b). In order to perform the Floquet analysis of the Raman laser homogeneous solution, we first computed numerically the spatial profiles of the pump and signal fields in the stationary state, by suppressing temporal modulations. After that, we repeated the Floquet analysis integrating numerically Eqs. (3) over one full cavity round trip.

In Fig. 4(b), the Faraday instability spectrum shows the presence of exponentially growing modulation sidebands. Consistently with these theoretical predictions, full numerical simulations of Eqs. (3) reveal that temporal Faraday patterns, consisting in a train of pulses, are indeed generated, owing to the self-induced Faraday instability. For a quantitative comparison with predictions of the linearized stability analysis, the repetition rate of the numerically generated pulse trains measured from the power spectrum peak is indicated by black dots in Fig. 4(b). As can be seen, the linear stability analysis provides a good estimation of the pulses repetition rate. The generated pulses are in general regular [see Fig. 4(d)], but sometimes are subject to transient instabilities and collisions; after those turbulent events the regular pulsation regime regenerates spontaneously [Fig. 4(e)].

We stress that periodic forcing in this case is not due to any action performed on the system from the external world, but it arises spontaneously, owing to the particular self-organization process of the field solutions, hence the denomination: self-induced Faraday instability. This feature distinguishes our laser system from previous and recent studies of dispersive or dissipative parametric instabilities [25,26,29-34,49,50], where the forcing is produced by a suitably designed periodic dispersive or dissipative landscape.

Conclusions. - In conclusion, our study sheds light on an up to now not considered case of self-induced PI, and on the associated pattern formation process, taking place in laser systems described by the universal CGLE, and its generalized versions. We have shown, based on realistic examples, that lasers with a suitable inhomogeneous spatial distribution of gain along the resonator can be the ideal platform for observing the predicted self-induced Faraday instability. The primary interest in the associated temporal pattern formation process based on Raman gain is linked to the possibility of exploiting the self-induced Faraday instability to design pulsed fiber laser sources operating at high repetition rates, widely tunable across the entire transparency window of optical fibers.

This project has received funding from the European Union's Horizon 2020 research and innovation program under the Marie Skłodowska-Curie Grant Agreement No. 691051. Authors acknowledge financial support from the Spanish Ministerio de Ciencia e Innovación, and the European Union FEDER through Project No. FIS201565998-C2-1-P. The work of S. W., S. V. S., and D. V. C. is supported by Ministry of Education and Science of the Russian Federation (Minobrnauka) (14.Y26.31.0017). A. M. P. acknowledges support from the ICONE Project through Marie Skłodowska-Curie Grant No. 608099.

Corresponding author. peregoa@aston.ac.uk

[1] T. B. Benjamin and J.E. Feir, The disintegration of wave trains on deep water. Part I. Theory, J. Fluid Mech. 27, 417 (1967).

[2] A. Hasegawa, Plasma Instabilities and Nonlinear Effects (Springer-Verlag, Heidelberg, 1975).

[3] K. Tai, A. Hasegawa, and A. Tomita, Observation of Modulational Instability in Optical Fibers, Phys. Rev. Lett. 56, 135 (1986).

[4] L. A. Lugiato and R. Lefever, Spatial Dissipative Structures in Passive Optical Systems, Phys. Rev. Lett. 58, 2209 (1987).

[5] F. T. Arecchi, G. Giacomelli, P. L. Ramazza, and S. Residori, Vortices and Defect Statistics In Two-Dimensional Optical Chaos, Phys. Rev. Lett. 67, 3749 (1991).

[6] K. Staliunas and V. J. Sanchez-Morcillo, Transverse Patterns in Nonlinear Optical Resonators, Springer Tracts in Modern Physics (Springer Verlag, New York, 2003), Vol. 183.

[7] M. Tlidi, K. Staliunas, K. Panajotov, A. G. Vladimirov, and M. G. Clerc, Localized structures in dissipative media: From optics to plant ecology, Phil. Trans. R. Soc. A 372, 20140101 (2014).

[8] M. Nakazawa, K. Suzuki, and H. A. Haus, The modulational instability laser-part I: Experiment, IEEE J. Quantum Electron. 25, 2036 (1989); M. Nakazawa, K. Suzuki, H. Kubota, and H. A. Haus, The modulational instability laser-part II: Theory, IEEE J. Quantum Electron. 25, 2045 (1989).

[9] M. Haelterman, S. Trillo, and S. Wabnitz, Dissipative modulational instability in a nonlinear dispersive ring cavity, Opt. Commun. 91, 401 (1992).

[10] M. Haelterman, S. Trillo, and S. Wabnitz, Additivemodulation instability ring laser in the normal dispersion regime of a fiber, Opt. Lett. 17, 745 (1992).

[11] M. Haelterman, S. Trillo, and S. Wabnitz, Hopf sideband bifurcation and chaos in fiber lasers with injected signal, Phys. Rev. A 47, 2344 (1993).

[12] P. Franco, F. Fontana, I. Cristiani, M. Midrio, and M. Romagnoli, Self-induced modulational-instability laser, Opt. Lett. 20, 2009 (1995).

[13] R. E. Kennedy, S. V. Popov, and J. R. Taylor, Ytterbium gain band self-induced modulation instability laser, Opt. Lett. 31, 167 (2006).

[14] E. Yoshida and M. Nakazawa, Low-threshold 115-GHz continuous-wave modulational-instability erbium-doped fiber laser, Opt. Lett. 22, 1409 (1997). 
[15] C. J. S. de Matos, D. A. Chestnut, and J. R. Taylor, Lowthreshold self-induced modulational instability ring laser in highly nonlinear fiber yielding a continuous-wave $262-\mathrm{GHz}$ soliton train, Opt. Lett. 27, 915 (2002).

[16] M. Quiroga-Teixeiro, C. Balslev Clausen, M. P. Sørensen, P. L. Christiansen, and P. A. Andrekson, Passive mode locking by dissipative four-wave mixing, J. Opt. Soc. Am. B 15, 1315 (1998).

[17] T. Sylvestre, S. Coen, Ph. Emplit, and M. Haelterman, Selfinduced modulational instability laser revisited: Normal dispersion and dark-pulse train generation, Opt. Lett. 27, 482 (2002).

[18] T. Sylvestre, S. Coen, O. Deparis, Ph. Emplit, and M. Haelterman, Demonstration of passive modelocking through dissipative four-wave mixing in fibre laser, Electron. Lett. 37, 881 (2001).

[19] H. Risken and K. Nummedal, Instability of off resonance modes in lasers, Phys. Lett. A 26, 275 (1968).

[20] R. Graham and H. Haken, Quantum theory of light propagation in a fluctuating laser-active medium, Z. Phys. 213, 420 (1968).

[21] E. M. Pessina, G. Bonfrate, F. Fontana, and L. A. Lugiato, Experimental observation of the Risken-NummedalGraham-Haken multimode laser instability, Phys. Rev. A 56, 4086 (1997).

[22] L. A. Lugiato, F. Prati, and M. Brambilla, Nonlinear Optical Systems (Cambridge University Press, Cambridge, England, 2015).

[23] M. C. Cross and P. C. Hohenberg, Pattern formation outside of equilibrium, Rev. Mod. Phys. 65, 851 (1993).

[24] M. Faraday, On a peculiar class of acoustical figures; and on certain forms assumed by a group of particles upon vibrating elastic surfaces, Phil. Trans. R. Soc. London 121, 299 (1831).

[25] C. Szwaj, S. Bielawski, D. Derozier, and T. Erneux, Faraday Instability in a Multimode Laser, Phys. Rev. Lett. 80, 3968 (1998).

[26] K. Staliunas, C. Hang, and V. V. Konotop, Parametric patterns in optical fiber ring nonlinear resonators, Phys. Rev. A 88, 023846 (2013).

[27] W. van Saarloos and J. D. Weeks, Faraday Instability of Crystallization Waves at the ${ }^{4} \mathrm{He}$ Solid-Liquid Interface, Phys. Rev. Lett. 74, 290 (1995).

[28] A. L. Lin, M. Bertram, K. Martinez, H. L. Swinney, A. Ardelea, and G. F. Carey, Resonant Phase Patterns in a Reaction-Diffusion System, Phys. Rev. Lett. 84, 4240 (2000).

[29] A. M. Perego, N. Tarasov, D. V. Churkin, S. K. Turitsyn, and K. Staliunas, Pattern Generation by Dissipative Parametric Instability, Phys. Rev. Lett. 116, 028701 (2016).

[30] M. I. Afzal, K. Alameh, and Y. T. Lee, Self-induced modulational-instability-assisted frequency comb generation using a passive fiber ring cavity having varying normal dispersion, IEEE J. Sel. Top. Quantum Electron. 20, 0900606 (2014).

[31] M. Conforti, A. Mussot, A. Kudlinski, and S. Trillo, Modulational instability in dispersion oscillating fiber ring cavities, Opt. Lett. 39, 4200 (2014).

[32] M. Conforti, F. Copie, A. Mussot, A. Kudlinski, and S. Trillo, Parametric instabilities in modulated fiber ring cavities, Opt. Lett. 41, 5027 (2016).
[33] F. Copie, M. Conforti, A. Kudlinski, S. Trillo, and A. Mussot, Dynamics of Turing and Faraday instabilities in a longitudinally modulated fiber-ring cavity, Opt. Lett. 42, 435 (2017).

[34] F. Copie, M. Conforti, A. Kudlinski, S. Trillo, and A. Mussot, Modulation instability in the weak dispersion regime of a dispersion modulated passive fiber-ring cavity, Opt. Express 25, 11283 (2017).

[35] K. Staliunas, S. Longhi, and G. J. de Valcarcel, Faraday Patterns in Bose-Einstein Condensates, Phys. Rev. Lett. 89, 210406 (2002).

[36] P. Engels, C. Atherton, and M. A. Hoefer, Observation of Faraday Waves in a Bose-Einstein Condensate, Phys. Rev. Lett. 98, 095301 (2007).

[37] H. Al-Jibbouri, I. Vidanović, A. Balaž, and A. Pelster, Geometric resonances in Bose-Einstein condensates with two- and three-body interactions, J. Phys. B 46, 065303 (2013).

[38] K. Krupa, A. Tonello, A. Barthélémy, V. Couderc, B. M. Shalaby, A. Bendahmane, G. Millot, and S. Wabnitz, Observation of Geometric Parametric Instability Induced by the Periodic Spatial Self-Imaging of Multimode Waves, Phys. Rev. Lett. 116, 183901 (2016).

[39] F. Matera, A. Mecozzi, M. Romagnoli, and M. Settembre, Sideband instability induced by periodic power variation in long-distance fiber links, Opt. Lett. 18, 1499 (1993).

[40] S. Wabnitz, Optical turbulence in fiber lasers, Opt. Lett. 39, 1362 (2014).

[41] S. M. J. Kelly, Characteristic sideband instability of periodically amplified average soliton, Electron. Lett. 28, 806 (1992).

[42] J. P. Gordon, Dispersive perturbations of solitons of the nonlinear Schrödinger equation, J. Opt. Soc. Am. B 9, 91 (1992).

[43] D. J. Jones, Y. Chen, H. A. Haus, and E. P. Ippen, Resonant sideband generation in stretched-pulse fiber lasers, Opt. Lett. 23, 1535 (1998).

[44] J. Javaloyes, T. Ackemann, and A. Hurtado, Arrest of Domain Coarsening via Antiperiodic Regimes in Delay Systems, Phys. Rev. Lett. 115, 203901 (2015).

[45] B. I. Shraiman, A. Pumir, W. van Saarloos, P. C. Hoenberg, H. Chaté, and M. Holen, Spatiotemporal chaos in the onedimensional complex Ginzburg-Landau equation, Physica (Amsterdam) 57D, 241 (1992).

[46] H. Chaté, Spatiotemporal intermitttency regimes of the onedimensional complex Ginzburg-Landau equation, Nonlinearity 7, 185 (1994).

[47] I. S. Aranson and L. Kramer, The world of the complex Ginzburg-Landau equation, Rev. Mod. Phys. 74, 99 (2002).

[48] D. V. Churkin, S. V. Smirnov, and E. V. Podivilov, Statistical properties of partially coherent cw fiber lasers, Opt. Lett. 35, 3288 (2010).

[49] N. Tarasov, A. M. Perego, D. V. Churkin, K. Staliunas, and S. K. Turitsyn, Mode-locking via dissipative Faraday instability, Nat. Commun. 7, 12441 (2016).

[50] A. M. Perego, High-repetition-rate, multi-pulse all-normaldispersion fiber laser, Opt. Lett. 42, 3574 (2017). 